\title{
A Multi-Pronged Approach to Adaptive and Context Aware Content Dissemination in VANETs
}

\author{
Joao M. Duarte • Eirini Kalogeiton • Ridha Soua • Gaetano Manzo • \\ Maria Rita Palattella • Antonio Di Maio - Torsten Braun • Thomas \\ Engel • Leandro A. Villas • Gianluca A. Rizzo
}

Received: date / Accepted: date

Abstract Content dissemination in Vehicular Ad-hoc Networks (VANETs) has the potential to enable a myr-

Joao M. Duarte

University of Bern, Switzerland, IC, University of Campinas, Brazil

E-mail: duarte@inf.unibe.ch

Joao M. Duarte

IC, University of Campinas, Brazil

Eirini Kalogeiton

University of Bern, Switzerland

E-mail: kalogeiton@inf.unibe.ch

Torsten Braun

University of Bern, Switzerland

E-mail: braun@inf.unibe.ch

Ridha Soua

SnT, University of Luxembourg, Luxembourg

E-mail: ridha.soua@uni.lu

Maria Rita Palattella

SnT, University of Luxembourg, Luxembourg

E-mail: mariarita.palattella@list.lu

Antonio Di Maio

SnT, University of Luxembourg, Luxembourg

E-mail: antonio.dimaio@uni.lu

Thomas Engel

SnT, University of Luxembourg, Luxembourg

E-mail: thomas.engel@uni.lu

Gaetano Manzo

HES-SO, Switzerland

E-mail: Gaetano.Manzo@hevs.ch

Gaetano Manzo

University of Bern, Switzerland

Gianluca A. Rizzo

HES-SO, Switzerland

E-mail: Gianluca.Rizzo@hevs.ch

Leandro A. Villas

IC, University of Campinas, Brazil

E-mail: leandro@ic.unicamp.br iad of applications, ranging from advertising, traffic and emergency warnings to infotainment. This variety in applications and services calls for mechanisms able to optimize content storing, retrieval and forwarding among vehicles, without jeopardizing network resources. Content Centric Networking (CCN), takes advantage of inherent content redundancy in the network in order to decrease the utilization of network resources, improve response time and content availability, coping efficiently with some of the effects of mobility. Floating Content (FC), on the other hand, holds potential to implement efficiently a large amount of vehicular applications thanks to its property of geographic content replication, while Software Defined Networking (SDN), is an attractive solution for the lack of flexibility and dynamic programmability that characterizes current VANET architectures. By implementing a logical centralization of the network, SDN enables dynamic and efficient management of network resources.

In this paper, for a few reference scenarios, we illustrate how approaches that combine $\mathrm{CCN}, \mathrm{FC}$ and SDN enable an innovative adaptive VANET architecture able to efficiently accommodate to intermittent connectivity, fluctuating node density and mobility patterns on one side and application performance and network resources on the other side, aiming to achieve high QoS. For each scenario, we highlight the main open research challenges, and we describe possible solutions to improve content dissemination and reduce replication without affecting content availability.

Keywords VANETs - Content Centric Networking · Content Dissemination · Floating Content - Software Defined Networking · Content Caching · Replication 


\section{Introduction}

Vehicular Ad-hoc Networks (VANETs) allow communications among vehicles and between vehicles and fixed infrastructure, aiming to support a wide range of services and applications to make travel experience pleasant, safe, and informed [1]. Applications envisioned for VANETs vary from traffic conditions and accident warnings to services such as live video streaming, live gaming, etc. The main technical challenges in VANET communications are related to the high dynamicity and volatility of the vehicular environment. Therefore, mechanisms for online adaptation of the network configurations to the wireless medium, to the highly varying inter-user distance and to node density, etc. are required. Though a lot of work has been done in proposing mechanisms for efficient content dissemination, it is still an open issue how to reliably support infotainment and other video streaming related applications with acceptable Quality of Service (QoS).

This paper describes a possible approach to tackle this issue, based on combining three paradigms: Content-Centric Networking (CCN), Floating Content (FC) and Software-Defined Networking (SDN). Content-Centric Networking [2] allows messages to be exchanged throughout the network based on their content and not on the location of the hosts. The CCN communication model is based on the exchange of Interest and Data messages and is supported by three main data structures: the Content Store (CS) to cache incoming content, the Pending Interest Table (PIT) to keep track of Interest messages and the Forwarding Information Base (FIB) to store communication interfaces. Figure 1 shows the basic architecture of a $\mathrm{CCN}$ node. In settings characterized by high node mobility and volatility, such as VANETs, CCN, due to its content centric but not host centric approach, may greatly increase the chance of delivering the requested content in case of disrupted links and frequent changes of network topologies [3]. However, adapting the routing decisions to such changes is critical to achieve a good balance between optimizing content delivery likelihood and resource utilization. In warning applications, data packets are usually small and can be disseminated between vehicles equipped with On-Board Units (OBUs) and between vehicles and infrastructure such as Road Site Units (RSUs) without significantly affecting the available communication bandwidth. For these types of services, the push-based communication approach, usually adopted in publish/subscribe applications, is well suitable. Therefore, Floating Content (FC), an opportunistic communication scheme, which supports infrastructure-less distributed content sharing over a given geographic area, could be a good candidate for the implementation of these services.

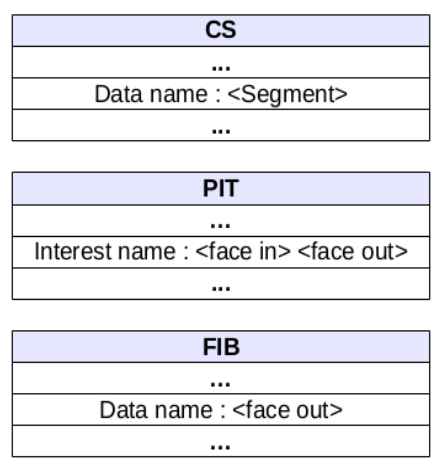

Fig. 1: Architecture of a CCN node

The basic formulation of FC is push-based and supports settings, in which a large fraction of nodes in a given area (the Anchor Zone, AZ) are interested in receiving small messages [4]. The idea is to store a given content in a spatial region without any fixed infrastructure, making it available through opportunistic communications to all users traversing that region. Whenever a node possessing a content is within the transmission range of some other nodes not having it, the content is replicated. When a node with the content moves out of its spatio-temporal limits, it deletes the content. The content may be available on a set of nodes and moves over time within the AZ, even after the node that has originally generated it has left the AZ. Thus, the content 'floats' within the AZ. The ratio between this set and the total amount of nodes inside the AZ is called Availability. This value influences the probability that a node can get the content during its sojourn, which is the Success Probability. Besides the advantages that both FC and CCN may bring in storing and disseminating content in VANETs, their performance could be improved by the coordination of a centralized entity that has global knowledge of the nodes mobility, their interest in a given content, as well as other informations. This entity can properly set FC and CCN parameters (e.g. AZ size, time to replicate content, strategy for content caching) and select which approach is more suitable for a given use case [5]. Initially designed for wired networks, Software Defined Networking (SDN) has also been recognized as an attractive and promising approach for wireless and mobile networks [6]. These types of networks can benefit from the flexibility, programmability and centralized control view offered by SDN, under different aspects, such as wireless resource optimization (i.e. channel allocation, interference avoidance), packet routing and forwarding in multi-hop multi-path scenarios as well as efficient mobility and network heterogeneity management. The pioneering SDN-based architecture for VANETs was proposed by $\mathrm{Ku}$ et al. [7], and afterwards enhanced $[8,9]$ by adding cloud and fog 
computing components. In a scenario with a variety of vehicular services with very diverse communication requirements, SDN holds the potential to improve the management of content delivery. A first Type-Based Content Distribution (TBCD) method was proposed in [10] to improve content caching and forwarding in an SDN-enabled VANET. TBCD adopts a push-and-pull approach for delivering content, based on the type of content, and on the number of users interested in it.

The contributions of the present paper, which extends our previous work [11], is threefold. First, it highlights the potentials of using SDN in VANETs as well as the relevant architectures that support SDN-enabled VANETs. This is of particular interest in understanding how $\mathrm{CCN}$ and FC could benefit from SDN features to optimize content dissemination in a network with intrinsic intermittent connectivity. Second, it analyzes how several factors, such as mobility patterns, infrastructure availability, network density, etc. can impact both CCN and FC based content dissemination. The purpose is to provide insights on how the control of these parameters could enhance content delivery probability. Finally, it investigates also how equipping vehicles with different technologies (e.g. Wi-Fi, LTE) might reduce content retrieval time.

The rest of the paper is organized as follows. Section 2 highlights SDN benefits envisioned for vehicular networks and studies pertinent architectures proposed in the literature for SDN-enabled VANETs. Section 3 describes how CCN and FC can be applied in vehicular network scenarios. Section 4 envisages the research areas where the use of an SDN controller may help improving the performance of $\mathrm{CCN}$ in vehicular networks. Section 5 illustrates how FC can benefit from some form of coordination among nodes, when implemented through a SDN controller. Finally, Section 6 concludes the paper.

\section{Software-Defined Wireless Networking for VANETs: Benefits and Architecture}

In this section we discuss the different architectures that were proposed notably for VANETs to overcome the standoff of SDN deployment in intelligent transportation systems. Before that, we briefly outline the several benefits brought by SDN in terms of flexibility, abstraction and programmability.

\subsection{SDN benefits in VANETs}

SDN, first proposed in [12], is a paradigm based on the decoupling of the data forwarding plane and the control plane. The latter, which represents the intelligence of the network, becomes logically centralized and programmable, whereas mobile nodes become simple forwarding devices. Hence, data plane devices are released from carrying out complex operations and their main function is restricted to only forwarding data according to forwarding policies prescribed by the controller. Hence, separating routing rules decisions from forwarding actions allows dynamic access and administration. This dynamicity is required in VANETs since the environment around vehicles, including weather conditions and neighbors, is continuously evolving and is shaping the behavior of vehicles. For instance, context- and location-driven routing updates requires dynamic policy programming. However, dynamic policy programming cannot be supported by the classical VANET architecture. Moreover, in future $5 \mathrm{G}$ networks, connected "things" should operate in a seamless way enabling a plethora of applications. Current VANET architectures cannot cope with this explosive growth in device and radio access heterogeneity. Stemming from these observations, we draw the main advantages expected from enabling SDN in VANETs:

- Flexible Network configuration: The controller can shape network behavior without going through hard-coding of switches. The desired behavior is translated into a set of rules that can be changed when necessary (e.g. prioritizing a specific data flow) according to requirements driven by VANET applications [13]. Hence the controller might have coarsegrained control over the whole network while forwarding nodes may have local intelligence and apply fine-grained decision policies.

- Efficient network resource management: Since, the controller (implemented for instance in an RSU) has a holistic view of the network such as available bandwidth, spectrum resources, shortest and fast routes, it can intelligently decide at which time, what type of traffic will use a specific channel to avoid congestion and which route the data flow should follow to meet delay, throughput and load balancing requirements. In absence of this intelligence, network resources could be jeopardized when the size of the network grows [14].

- Network heterogeneity support: In future 5G networks, vehicles will be interconnected with a huge number of devices on top of several network technologies. For instance, they will collect data from sensors deployed on streets, using different lowpower network technologies (e.g. IEEE802.15.4 for short-range, or LPWAN for long range). Besides, vehicles are envisioned to communicate also with cellular networks for bandwidth demanding traffic. Subsequently, vehicles are involved in a myriad of communications that are not straightforward 
to support. SDN can overcome the heterogeneity of devices by seamlessly abstracting their differences with a unified managing control logic. This centralized logic allows the discovery of devices and their access to services in an abstract way. Moreover, SDN can implement smart routing and traffic steering over multiple network technologies to reduce packet delivery time and increase throughput and reliability [13].

\subsection{SDN architecture for VANETs}

Figure 2 illustrates a typical SDN-enabled architecture for VANETs, which is in line with those that have been proposed so far in literature $[7,13]$. We now describe its main constituent parts:

- Data plane: Both stationary (RSUs, BSs) and mobile nodes (vehicles) constitute the data plane. Likewise for other elements of the data plane, vehicles could be equipped with multiple radio technologies for control and data exchange.

- Control plane: The control plane is logically centralized. The controller collects the status of vehicles, RSUs, and BSs, which are abstracted as SDN switches via IEEE 802.11 p or Wi-Fi beacons. The status includes vehicle location, speed, direction, neighbor information and vehicles in coverage range of RSUs or BSs. Basically, a RSU Controller (RSUC) communicates with data plane elements and instructs them on forwarding rules or resource allocation strategies based on collected status and current context of vehicles. In a fully centralized operative mode (single controller), the architecture is prone to the well-known drawbacks of traditional wired SDN (e.g. presence of single points of failure, loss of connectivity between RSUC and data plane, etc). Therefore, some distributed operative modes were proposed to ensure scalability. A large VANET is partitioned into clusters and each cluster is managed by its own controller. However, this zoning [14] is not adequate for computing optimal routes for packet delivery. In addition, controllers managing each cluster require tight coordination to ensure performance across zones. An alternative is the hybrid architecture, in which the control of the network is shared between the RSUC and the local SDN agents that are installed in certain RSUs and vehicles. The controller sends an abstract policy to RSUCs or BSs and then RSUs decide about specific behaviors depending on their local knowledge. An illustration of rule forwarding operation is depicted in Figure 2. The intelligence as well as the complexity of network control are split between the RSUC and the distributed data forwarding elements. Consequently, hybrid architecture is more suitable to break scalability barriers.

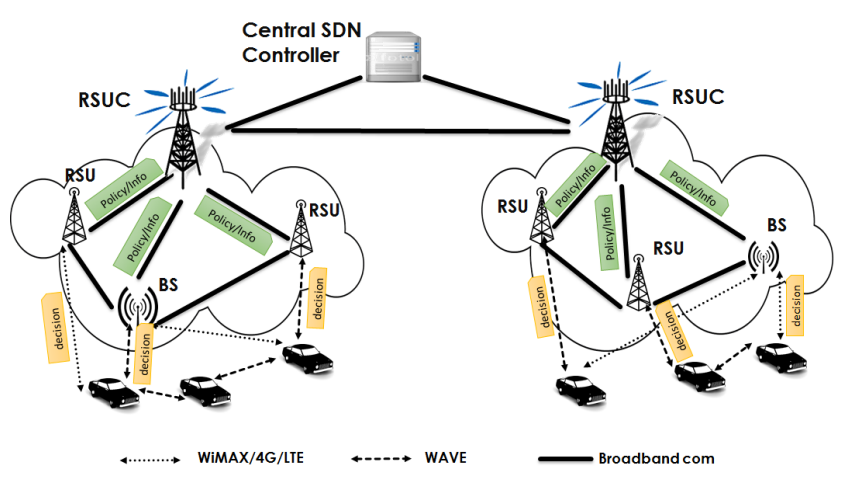

Fig. 2: Hybrid architecture for SDN enabled VANETs

- Communication interfaces: To ensure that instructions are received by data plane elements, control plane and data plane communicate via a unified SouthBound Interface (SBI). One of the widely used SBI is OpenFlow [12], which is mainly conceived for wired networks. In VANET scenarios, OpenFlow should be redesigned to adapt to nodes mobility and the intermittent connectivity in the data plane. Recently, an extension of OpenFlow was proposed in [13], allowing vehicles to report positions and velocity. Nevertheless, no technical details on how this extension is implemented are provided.

\section{Content Dissemination in VANETs}

The unique characteristics of VANETs and the heterogeneity in communication requirements of vehicular applications make it hard to find a general dissemination mechanism that performs well in all situations. Some related works already proposed CCN modifications to adapt the paradigm to VANET features. Goals were to decrease packet forwarding load and deal with difficulties in maintaining Forwarding Information Base (FIB) entries. For instance, Amadeo et al. [15], introduced an additional field in the Data message to help requesters in selecting the best content provider. To increase content availability, authors in [16] propose that each vehicle caches all received Data and forwards all Interest messages to all available interfaces. However, increasing message size and forwarding multiple Interest messages for a given content increase traffic load.

\subsection{Content Dissemination based on CCN and FC}

The coupling between $\mathrm{CCN}$ and FC can provide suitable adaptive solutions for content dissemination over VANETS. When content objects are bound to a specific geographic region, and stored on all (or a subset of) nodes within the region itself, FC can play a key role for 
improving CCN. By replicating content, FC introduces redundancy, which can be useful when nodes leave the area or lose connectivity with their neighbors and hence do not make content permanently available. Moreover, FC can make searching and finding content more efficient, if the geographic area of the floating content can be derived from the content name. Normally, to retrieve content, CCN requesters issue Interest messages including a content name describing the requested content. To ensure that the content bound to a geographic area is found, the content name can be mapped to that area. Consequently, an Interest message is forwarded to that geographic area to meet the requested content. Mapping can be supported by either having a geographic ID in the content name or by having an intermediate node, which translates the original content name into a geographic name. After the Interest has met a content source, a Data message including the content is sent back to the requester based on the stored information in the PITs.

\subsection{Adaptive Mechanisms for Content Dissemination in Vanets}

Context parameters such as type of mobility, network density, availability of RSUs along the path, propagation models, etc. influence the operation of content dissemination and retrieval mechanisms in VANETs and should be taken into account. Therefore, we argue that a self-adaptive and context-aware mechanism for content dissemination in VANETs, able to dynamically adjust its network configurations, routing decisions, and anchor zone (AZ) characteristics is crucial and has the potential to increase content delivery probability.

Furthermore, network configurations including transmission power and directions, data rate, packet lengths, etc. should be adapted to a set of specific parameters such as the condition of the transmission channel, the distance between nodes, node density and propagation models. For instance, in highways, straight-line propagation may be observed while within a city obstacles such as buildings and other objects may cause reflexions, deflections, refractions etc. In rural areas different models may coexist.

Based on targeted performance metrics, routing and forwarding decisions should be programmed and updated. For example, when nodes are located close to each other, a single transmission performed by the node with better reachability may be enough to disseminate content to all its neighbors. On the other side, when nodes are located far away from each other, a multi-hop approach that minimizes the number of transmissions and hence delay is needed. AZ shapes and sizes can be adapted according to the characteristics of each applica- tion and current node density. For example, information from a road condition warning application may be important for vehicles within a radius of a few hundreds of meters while information from a traffic jam application may be useful for vehicles within several tens of kilometers. In a highway scenario, a rectangular AZ shape is more appropriate, whereas in a city center, square or circular AZ shapes may reach more vehicles.

\subsection{Content Distribution and Mobility}

In this section, we discuss the various issues, parameters, and situations that can impact the performance of content distribution mechanisms based on CCN and FC due to the mobility in VANETs. Efficient control of these parameters is essential to maintain high content delivery and retrieval probabilities while reducing the number of Interest and Data messages collisions, retransmissions, duplicates and hence delays.

\subsubsection{Mobility patterns}

Mobility patterns are a decisive feature for content delivery probability. The performance of dissemination mechanisms may vary according to source and receiver mobility. We distinguish three cases in the following:

- No mobility of the source and the receiver: In cases where there is no mobility of either source and receiver, standard CCN Interest/Data exchange based on CS, PIT and FIB can be used.

- Mobility of the receiver: When receiver mobility is present the following cases can happen:

(i) Low speed: In case of low receiver speed, standard CCN Interest/Data exchange based on CS, PIT and FIB still can be used, if the mobile receiver is still in reach of the node to whom it sent its Interest message after one Interest/Data round-trip-time. This can be estimated by considering distance between nodes, RSSI values, node speeds and directions, and RTT measurements.

(ii) High speed: In case of high receiver speed, the soft state established by Interests in CCN routers can not be used since the data traveling back following the PIT entries may not reach the requester anymore. Possible solutions are:

In case of accurate mobility prediction, e.g. by navigation systems, public transport usage etc., and availability of RSUs along the path: The task of content retrieval is delegated to agents (see Subsection 3.5). Alternatively, the receiver can indicate its trace and estimated future positions and then, the source sends content to the estimated position. This might require accurate mobility prediction and possibly tracking of mobile nodes. 
In case of inaccurate mobility prediction or unavailability of RSUs along the path: Receivers can indicate a next destination in their path where to receive the content. Then, sources can send the content towards that destination and make the content float. The receiver can then request it from there or from the original source or has to indicate a new location, e.g. if it changes its path.

- Mobility of the source: Source mobility can impact drastically the probability that an Interest message sent by requesters will meet with at least one node able to send back the requested data. For this, the following possibilities may be of use:

(i) The source can create an AZ for content that is relevant to a certain area to store and advertise it. When leaving the created AZ, vehicles may then replicate the content in order to maintain it in that area for a certain time to live (TTL), established by the original source node.

(ii) Mobility prediction can be used to estimate the path of the source and forward Interest messages towards the estimated source position.

\subsubsection{Network density}

Network density can range from dense networks, where a huge number of nodes is available on a specific area, to sparse networks, where only few nodes are present. In dense networks it is important to use efficient duplicate suppression mechanisms $[17,18]$ to limit the amount of concurrent transmissions and thus decrease the probability of collisions.

As the number of nodes in the network decreases and the network becomes sparse, duplicate suppression mechanisms become less crucial. However, in these cases the probability of network disruptions increases and efficient Delay-and-Disruption Tolerant Network (DTN) routing to ensure communication is required. Network disruptions can also happen in dense networks, e.g. in case of large unpopulated areas such as lakes, parks, etc.

\subsubsection{Network partitions and temporal fragmentation}

Network partition occurs when the number of vehicles in the AZ is not sufficient to guarantee data dissemination between vehicles. Temporal network fragmentation on the other hand refers to situations where communications between vehicles are disrupted due to packet losses, collisions or obstacles in the communication path between a group of vehicles. The caching property of CCN may be an efficient solution for these issues by supporting the implementation of the so called "storecarry-forward" mechanism, where vehicles can carry a requested content and deliver it towards the requester when an opportunistic link is available.

\subsection{Multi-hop Content Retrieval}

In several vehicular scenarios, some vehicles moving into a given direction, along a main road or a highway, may be interested in retrieving content (e.g. videos) containing information about a distant location. For instance, fire trucks would be interested in receiving in advance videos of a fire to which they are heading, in order to estimate fire parameters such as severity, size, growth rate, etc., before reaching the intended location. In these cases, information received from videos can proactively support efficient planning of the work to be carried on.

To request a video, a requester vehicle may send an Interest message towards the location where the video should be collected. However, getting immediately the requested video is not straightforward as one single video is likely to be segmented into multiple chunks and therefore several packets may be transmitted in a reduced time frame to deliver the whole video. On the other hand, the distance between the location of the requester at the time of issuing the Interest message, and the location where the video should be collected, is likely higher than the communication range of vehicles (i.e. few hundreds of meters if IEEE802.11p based communication mechanisms such as [19] are used). Thus, a multi-hop mechanism for dissemination of Interest and Data messages is proposed. Neighbor vehicles are used as intermediate nodes. However, only a subset of the intermediate nodes, may be selected to retransmit an Interest message in order to eliminate unnecessary retransmissions.

\subsubsection{Our approach:}

We propose that Interest messages carry the type, name and location of the requested content (e.g. video/ fire /latitude/longitude). Intermediate nodes when receiving an Interest message will perform a lookup in their CS and if the requested content is not available they will schedule the retransmission of the Interest message based on an Interest timer. The values of Interest timers are calculated, taking as inputs the distance from the previous sender and the expected time for which the vehicle will remains in the specific point of the road. In this case, vehicles that are located farther away from the previous sender and that will stay in the road for long time will have shorter Interest timer periods.

When the Interest timer expires, the scheduled Interest message is retransmitted and the PIT table is updated by adding an entry related to the requested 
data. The remaining intermediate nodes, when perceiving that the Interest message has been retransmitted by another vehicle (i.e. the vehicle with shorter timer duration), will cancel their scheduled retransmission.

When intermediate nodes receive an Interest message and find a copy of the requested data in their CS, they automatically schedule the transmission of the Data message, using a Data timer (Data timers are shorter than Interest timers) and cancel the dissemination of the Interest message. Among the intermediate nodes that contain the requested data the one located closer to the requester node will have a lower Data timer value and will be selected to forward the Data message and therefore cancel both Interest and Data messages scheduled by other nodes for that particular piece of data.

If none of the intermediate nodes find a copy of the requested data in their $\mathrm{CS}$, the Interest message will eventually reach its intended destination. In this situation, the vehicle geographically located closer to the destination given in the Interest message will collect the video and send it back towards the requester node. When intermediate nodes receive a Data message containing a piece of video, the data is first added to the CS. Then a PIT lookup is performed and if an entry related to that data is found the Data message is sent towards the requester and the PIT entry is eliminated.

\subsection{Agent-Based Content Retrieval (ABCR)}

Another approach for requesting and receiving content is based on using agents. Requesters can delegate content retrieval to one or more agents [3] and retrieve from them the content later. A requester broadcasts an Interest to potential agents, which respond by Data messages describing their offer to retrieve content on behalf of the requester. The requester then confirms the agent selection. Later, when the requester meets the agent again, it can retrieve the content using traditional CCN message exchange.

In a VANET, a user might like to retrieve a content object from a location that is not in range, and where the vehicle is not scheduled to go. By letting requester and agent know their respective typical or expected paths, the content source could select the right agent (the one with the right path) for sending back the content object. Here we assume that the content source knows the path of the requester and of the candidate agents for carrying the content and that the content source will give information about the path of the requester to the agent carrying the content. Uncertainty in space and time can make the rendezvous hard to be implemented. To face this issue, the agent carrying the content could implement an AZ, in which the desired content floats and that the requester will traverse with high likelihood.

Agents can also be used to come up with a solution for receiver mobility without the need to modify $\mathrm{CCN}$. To address receiver mobility, a mobile requester intending to visit an indicated area can delegate content retrieval to an agent located in the indicated area. The agent requests the information from the content source and disseminates it using FC mechanisms in the indicated area. The mobile receiver then picks up the content from the agent or from another FC node when visiting the indicated area.

\subsection{FC-assisted, Geographic Content Centric (DTN) Routing}

In settings where volatility makes it unfeasible or inefficient to maintain a path between content requester(s) and content source, FC may be used to support socalled geographic content centric routing. We assume that all nodes and content objects can be identified uniquely, and that nodes know their location and future trajectory with some uncertainty. Such knowledge could be derived from past spatio-temporal patterns. By letting each node share through FC its own data on network state (e.g., node positions and trajectories, node content), FC can be used to build at each node a common, shared representation of the network and its evolution over time. Such representation could be used to implement various strategies for DTN routing.

As a complement, when network density is sparse and/or the mobility of nodes does not allow to build a stable path for content dissemination, Interest packets could be made to float in an area around the originator node. The floating content would be used as a "fishing net" to improve the chance of finding content among passing nodes. As a result, each node within an area would then maintain and share a table (i.e., floating PIT), describing a list of requested content objects. For each requested content object, the table stores a list of requesters. When meeting a node with one or more of the requested content objects, those could be forwarded to the requesters using some form of DTN routing scheme, possibly using the floating network state.

\section{SDN Support for CCN in VANETs}

In this section, we detail how content forwarding and caching could be enhanced by coupling SDN and CCN in VANETs. 


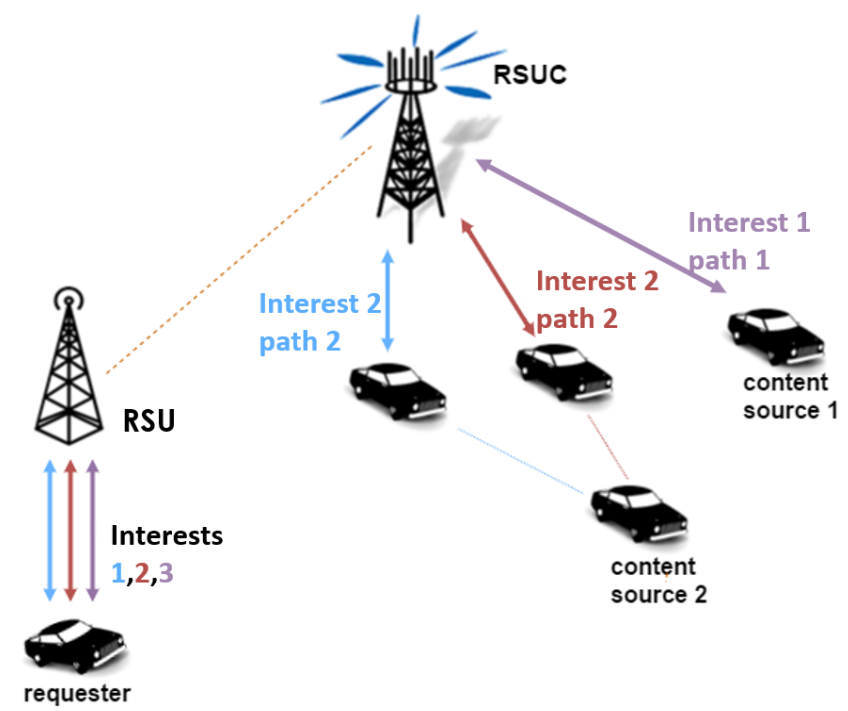

(a)

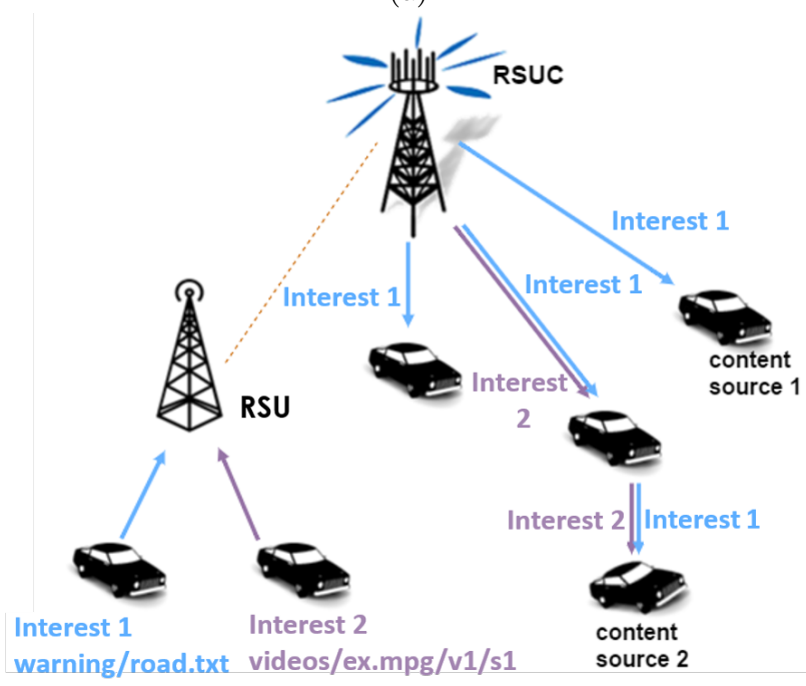

(b)

Fig. 3: SDN support for CCN forwarding in VANETs: (a) Forwarding of Interests along different paths, (b) Different forwarding strategies based on Interest names

\subsection{SDN for CCN Forwarding and Broadcasting}

In large-scale networks the size of FIB tables on each CCN node can easily increase due to the large amount of exchanged content and the large number of content sources. Alternatively, CCN routers can broadcast Interest messages to neighbor nodes to request content. Subsequently, the possibility of congestion in paths through the network can be very high. There have been several attempts to encounter these problems; some of them try to identify a path between a requester and a content source and store this path for future references $[20,21]$. A similar approach has been adopted also in CCVN [15], where messages are broadcast in the entire
VANET, but in case of collisions they are re-broadcast according to the distance from previous senders. To improve content retrieval, in [22] authors suggest to utilize the different interfaces of a CCN node to transmit Interests simultaneously through multiple interfaces. For the same aim, Udugama et al. [23] propose to split the Interest messages of the same request and send them through multiple paths at the same time.

An SDN controller, with its centralized view of the network, can support the design of more efficient CCN forwarding strategies. For instance, a controller can be responsible for selecting unicast paths between requester and content source in a given cluster of the network [24]. To integrate SDN into CCN networks, Charpinel et al. [25] proposed a CCN controller that has a complete view of the network. It defines the forwarding strategy, and installs forwarding rules in the FIBs of CCN routers. In every $\mathrm{CCN}$ router, there is a Cache Rules Table (CRT). The CCN controller sends cache rules (replacement policies) to the CCN switches, which are stored in the CRT. The CCN router caches the content according to the rules that exist in the CRT. The presence of a centralized controller could be further exploited to improve CCN performance. For instance, an SDN controller can determine the number of chunks to transmit through a face and send them through multiple faces simultaneously [26].

\subsubsection{Our approach}

As shown in Figure 3a, SDN could support the set up of multi-path communication. When a vehicle sends or receives a message (Interest or Data), it could send (or receive) it through multiple (redundant) paths. An SDN controller could discover several paths that satisfy Interest messages of the same request. Then, the controller sends Interest messages through those paths in parallel to retrieve Data much faster.

In addition to path diversity, each vehicle could be equipped with diverse radio communication technologies. For instance, a vehicle could have two or more Wi-Fi interfaces, together with LTE. Thereby, the requester could send two or more Interest messages simultaneously to a local SDN controller [27]. Subsequently, the controller could respond at the same time to all these requests, since it could perform a multi-path discovery mechanism. Hence, this multi-radio concept would optimize network resources and achieve shorter content retrieval time. For instance, as shown in Figure $3 \mathrm{a}$, a requester sends three Interests to the SDN controller through three Wi-Fi interfaces. Afterwards, the controller that has already discovered available content sources (in the previous step) sends these Interests on these multiple paths. Finally, the SDN controller 
sends the retrieved Data back to the requester through the same interfaces.

In a CCN-based VANET, several applications will generate requests with different names. The network must treat these requests in a different way according to application requirements. For instance, a safety application generating warning messages should forward these messages to the entire network. On the contrary, an entertainment application sending video requests should forward these messages through an appropriate face in order to find a content source. An SDN controller could install such forwarding rules to make sure that each message is treated differently, according to its name (Figure $3 \mathrm{~b}$ ). The overall geographic area is divided into clusters, according to the location of the RSUCs. For each of these clusters, the RSUC is responsible for orchestrating and managing network resources. Dividing the network into clusters ensures more control and less complexity for network administration. Besides, vehicles are considered as data mules: they perform storecarry-forward, enabling information to travel through the different clusters of the network. When a vehicle is between distinct RSUC areas, a push approach for transferring warning Data messages to other vehicles can be used.

Obviously, RSUCs could communicate with each other by a wired connection. Bloom Filters could be used to advertise and exchange information between RSUCs and hence to populate the FIB tables of nodes [28]. The local RSUC that is responsible for each geographic area, in addition to the forwarding and the optimization of resources, could potentially push messages to vehicles These messages could be advertisements, safety messages or even local offers from the area. Furthermore, vehicles equipped with different radio technology interfaces select the appropriate technology for transmitting each flow based on some QoS metrics. For example, warning messages could use LTE to guarantee low latency and the high message delivery rates required by safety applications.

\subsection{SDN for CCN Caching}

Caching enhances content discovery, retrieval and delivery in VANETs by providing multiple sources (caches) of the content. However, the explosion of infotainment applications with their ubiquitous replicas creates an increasing demand for the scarce spectrum in VANETs. Basically, caching is coupled with two fundamental questions: what content to cache and where to cache it.

Caching all content along the delivery paths as suggested in [29] may cause serious performance degradation. Even if the cache size is not a major concern in VANETs, it is not obvious if this cache can keep up the pace with the increasing scale of multimedia content distribution over VANETs. For these reasons, it was proposed in $[30,31]$ to cache only popular content (i.e. content which has been requested a number of time equal or larger than a fixed Popularity Threshold, PT). Other works rely on the user interest for deciding what content to keep [32] (i.e. nodes will cache only the content they are interested in).

Concerning where to cache, one trivial solution is to cache replicas in every vehicle. The short-lived nature of links in VANETs, coupled with the time and spacerelevant nature of content, accentuates the concern about the selection of relevant nodes that can cache specific content. Therefore, most new caching schemes attempt to reduce caching redundancy by only selecting a subset of nodes in the delivery path. This subset of nodes has high probability to get a cache hit and hence nodes in this subset are called "central" nodes or influential nodes. Centrality-based solutions for node selection were proposed in the context of static networks. However, their extension to highly mobile networks, such as VANETs, is a thorny problem as centrality is not trivial[33]. Recent works [34,35] focus on the social aspect of caching. Users in the same social space are likely to request the same content. Hence, the content is proactively pushed to the cache of users' proximate neighbors. The definition of these proximate neighbors is challenging. While in [34] proximate nodes are 1-hop neighbors, authors of [35] define these nodes as the nodes having a specific ratio of common content items with the user.

\subsubsection{Our approach}

An SDN controller could instruct Content Providers $(\mathrm{CP})$ to trigger on-demand caching when popular content has been identified. In the same way, the SDN controller could fix the value of the PT according to different metrics. Moreover, the SDN controller, being the owner of the caching logic, will determine which vehicles should store replicas of the popular content at each period of time. These vehicles are called Content Cachers (CC). This centralized caching logic helps authorities to program and deploy the desired caching behavior.

Figure 4 illustrates an example of our proposed SDN-based caching approach in VANETs. Two RSUs are orchestrated by one RSU Controller (RSUC). Each $\mathrm{RSU}$ is covering a specific geographical area on a highway. The RSUC fixes the PT and the set of influential vehicles [36] in the zone. The set of influential nodes (i.e. nodes with better reachability than others) is timevariant since vehicles can enter and leave the network frequently. Therefore, the RSUC should periodically (or when needed) notify vehicles in the network about the updated set of influential nodes. Once the number of 


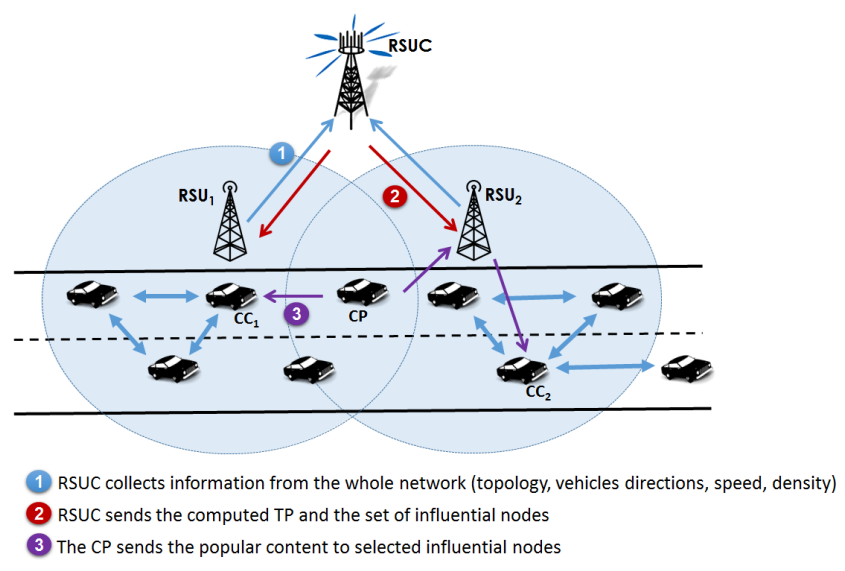

Fig. 4: SDN-based caching approach in VANETs.

requests for the content produced by the $\mathrm{CP}$, reaches the PT, the RSUC will trigger its caching spreading strategy. The popular content will not be sent to the immediate 1-hop neighbors of CP like in [34], but will be sent to the influential vehicles (as shown in Figure 4, $C C_{1}$ and $C C_{2}$ ). This mechanism avoids overloading the network with replicas of the same content. The CP is aware of the set of influential nodes and sends them accordingly the replicas. While $C C_{1}$ is a 1-hop neighbor of the $\mathrm{CP}, C C_{2}$ is out of its transmission range. Therefore, the content is sent first to $R S U_{2}$ and then $R U_{2}$ forwards the popular content to $C C_{2}$. Vehicles interested in the content of $\mathrm{CP}$ can ask $C C_{1}$ or $C C_{2}$ to retrieve it. Thus, vehicles far away from the CP (the node that generated the original content) can get replicas by asking the nearest $C C$.

\section{SDN support for FC in VANETs}

In a real scenario, determining the minimal size and the appropriate shape of the AZ to ensure high performance for FC (in terms of mean success probability, for instance) is a complex task. Authors of [37] have proven that the ratio between the $\mathrm{AZ}$ radius and the communication range impacts information availability. Authors of [38] provide the criticality condition, under which the content floats indefinitely over time with very high probability. All such results are closely tied to a specific mobility model, and in particular, they depend heavily on density, spatial distribution, speed and transmission range of nodes.

In addition, in the basic formulation of FC, content replication within the $\mathrm{AZ}$ does not take into account neither the popularity of the content nor the size of the set of nodes carrying the content. This is critical for VANETs, where a massive number of messages increases both spectrum congestion and management challenges.

\subsection{Our approach}

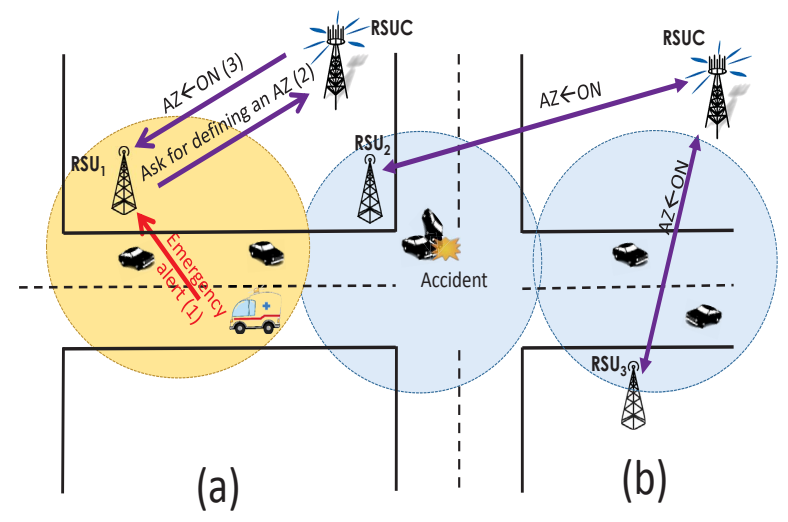

Fig. 5: (a) SDN-based FC activation, (b) AZ reshaping in an accident intersection scenario

There are two main ways to estimate node density and AZ size and shape: centralized and distributed ways. In the former one, a node in isolation and possessing needed information defines the AZ. In contrast, in the distributed way, nodes in a cooperative way, collect mobility information from their surrounding vehicles. This approach provides more accurate estimation of parameters such as density and average speed of nodes but requires longer time to converge. An attractive solution is to use a hybrid approach: the infrastructure (i.e. static nodes) is used as support for FC, thereby increasing the quality of estimation of parameters and thus achieving a balance between message replication ratio and the desired probability of success delivery.

To evaluate the success probability, several approaches were proposed in the literature. A very first approach is modeling the AZ range in order to obtain a certain success probability (i.e. QoS) considering all the other parameters (node density and spatial distribution, node speed distribution, and transmission range) fixed. Taking into account a circular AZ, different studies provide a well-posed success probability formula correlating with AZ radius. Some mobility parameters are independent from the mobility model chosen (e.g. node density). The higher node density is, the higher is the success probability, considering all the other mobility parameters fixed. Finally, setting the $\mathrm{AZ}$ radius could be done according to the node density (values defined by the scenario considered). This 
reshape could be achieved through infrastructure support (e.g. SDN controller, which collects mobility information such as speed and position of the vehicles in the coverage range). Therefore, an efficient anchor zone could be installed at the desired location. In a centralized approach, RSUs are in charge of setting the range of the AZ. Even if the coverage of RSUs is large, it may happen that the covered area does not match with the area of interest. Therefore a distributed approach may be more suitable.

In environments with highly varying density, SDN can ensure better persistence and availability of floating content by accurately tuning the AZ size. This online adaptation of AZ saves network resources. Figure 5(a) shows a use case where an AZ is created on demand. An emergency vehicle enters the zone covered by $R S U_{1}$ where there is no pre-established AZ. The emergency vehicle asks $R S U_{1}$ to define an $\mathrm{AZ}$. Then the request is forwarded by $R S U_{1}$ to the RSUC, which sends back the parameters of the AZ (shape, size, lifetime) based on the content lifetime, density of nodes and mobility patterns. SDN can also be involved in reshaping an existing AZ. One interesting use case is information dissemination in an intersection, which is a highly localized area as depicted in Figure 5(b). To ensure that the content is floating outside the AZ defined by the intersection, one possible solution would be to reshape the $\mathrm{AZ}$ and make it larger. Even if this can be done without infrastructure through a distributed approach, this latter does not guarantee a convergence to the desired results. However, as argued in [4], the bigger the AZ size is, the lower is the success probability (i.e. probability for a node entering in $\mathrm{AZ}$ to get the content), if node transmission ranges and density are fixed. Hence, the RSUC will define a new AZ based on its global knowledge of the network. Content replication can also be supported by infrastructure that provide an optimization of the estimation. The RSUC can define, through information collected from RSUs, the floating content priority. Subsequently, a node can decide to receive and/or ignore content, based on a priority rule that can take into account the content lifetime, number of nodes replicating the content, and the intermittent nature of the connections. Thus, the SDN controller implemented in RSUC could figure out the content popularity and decide with which frequency the content is replicated.

\section{Conclusions}

Improving the probability of success in delivery and minimizing content retrieval time without jeopardizing network resources are thorny problems in vehicular networks, where V2V and V2I connectivity are intermittent and short-lived. In this work, we have identified
CCN and FC as two suitable enablers for improving content storing, dissemination, and forwarding in future VANETs. Both should be adapted to the high dynamic and volatile network environment. However, current VANET architectures suffer from lack of flexibility and dynamic programmability. SDN, which decouples the control plane and data plane, is on the forefront of potential enablers of future VANETs, where routes can be dynamically configured at a logical centralized controller, but not at each hop as in classical VANETs. We discussed the benefits of applying SDN in VANETs and the features of SDN-enabled VANETs architectures. We investigated also how SDN could provide support for CCN and FC (e.g. to select, according to node mobility, the best paths to forward data packets, the influential nodes where the content should be cached, or to activate the AZ where the content should float).

\section{Acknowledgments}

This work was undertaken under the CONTACT project, CORE/SWISS/15/IS/10487418, funded by the National Research Fund Luxembourg (FNR) and the Swiss National Science Foundation (SNSF) project no. 164205. The authors also would like to thank The Coordenação de Aperfeiçoamento de Pessoal de Nivel Superior (CAPES) and the São Paulo Research Foundation (FAPESP) for the financial support by grant 2016/09254-3.

\section{References}

1. F. Cunha, L. Villas, A. Boukerche, G. Maia, A. Viana, R. A. F. Mini, and A. A. F. Loureiro. Data communication in vanets: Protocols, applications and challenges. Ad Hoc Networks, 44:90-103, 2016.

2. V. Jacobson, D. K. Smetters, J. D.Thornton, M. F. Plass, N. H. Briggs, and R. L. Braynard. Networking named content. In Proceedings of the 5th international conference on Emerging networking experiments and technologies, pages 1-12. ACM, 2009.

3. C. Anastasiades, T. Schmid, J. Weber, and T. Braun. Information-centric content retrieval for delay-tolerant networks. Computer Networks, 2016.

4. S. Ali, G. Rizzo, V. Mancuso, and M. A. Marsan. Persistence and availability of floating content in a campus environment. In 2015 IEEE Conference on Computer Communications (INFOCOM), pages 2326-2334, April 2015.

5. G. Rizzo, M. R. Palattella, T. Braun, and T. Engel. Content and context aware strategies for qos support in vanets. In 2016 IEEE 30th International Conference on Advanced Information Networking and Applications (AINA), pages 717-723, March 2016.

6. M. Yang, Y. Li, D. Jin, L. Zeng, X. Wu, and A. V. Vasilakos. Software-defined and virtualized future mobile and wireless networks: a survey. Mobile Networks and Applications, 20(1):4-18, 2014. 
7. I. Ku, Y. Lu, M. Gerla, R. Gomes, F. Ongaro, and E. Cerqueira. Towards software-defined vanet: Architecture and service. Conference: Annual Mediterranean Ad Hoc Networking Workshop MEDHOCNET, 2014.

8. M. A. Salahuddin, A. Al-Fuqaha, and M. Guizani. Software-defined networking for rsu clouds in support of the internet of vehicles. Internet of Things Journal, IEEE, 2(2):133-144, 2015

9. N. B. Truong, G. M. Lee, and Y. Ghamri-Doudane. Software defined networking-based vehicular adhoc network with fog computing. In Integrated Network Management (IM), 2015 Conference. IFIP/IEEE, 2015.

10. Y. Cao, J. Guo, and Y. Wu. Sdn enabled content distribution in vehicular networks. In Innovative Computing Technology (INTECH), 2014 Fourth International Conference on, pages 164-169. IEEE, 2014.

11. R. Soua, E. Kalogeiton, G. Manzo, J. M. Duarte, M. R. Palattella, A. Di Maio, T. Braun, T. Engel, L. A. Villas, and G. A. Rizzo. Sdn coordination for ccn and fc content dissemination in vanets. 2016.

12. N. McKeown, T. Anderson, H. Balakrishnan, G. Parulkar, L. Peterson, J. Rexford, S. Shenker, and J. Turner. Openflow: enabling innovation in campus networks. ACM SIGCOMM Computer Communication Review, 38(2):69-74, 2008.

13. Z. He, J. Cao, and X. Liu. Sdvn: enabling rapid network innovation for heterogeneous vehicular communication. IEEE Network, 30(4):10-15, July 2016.

14. M. Abolhasan, J. Lipman, W. Ni, and B. Hagelstein. Software-defined wireless networking: centralized, distributed, or hybrid? IEEE Network, 29(4):32-38, July 2015.

15. M. Amadeo, C. Campolo, and A. Molinaro. Enhancing content-centric networking for vehicular environments. Computer Networks, 57(16):3222-3234, 2013.

16. G. Grassi, D. Pesavento, G. Pau, R. Vuyyuru, R. Wakikawa, and L. Zhang. Vanet via named data networking. In Computer Communications Workshops (INFOCOM WKSHPS), 2014 IEEE Conference on, pages 410415. IEEE, 2014.

17. A. T. Akabane, L. A. Villas, E. Madeira, and M. Roberto. An adaptive solution for data dissemination under diverse road traffic conditions in urban scenarios. In 2015 IEEE Wireless Communications and Networking Conference $(W C N C)$, pages 1654-1659. IEEE, 2015.

18. L. Villas, A. Boukerche, G. Maia, R. W. Pazzi, and A. A. F. Loureiro. Drive: An efficient and robust data dissemination protocol for highway and urban vehicular ad hoc networks. Computer Networks, 75:381-394, 2014.

19. E. Donato, G. Maia, J. M. Duarte, A. A. F. Loureiro, E. Madeira, and L. Villas. Presync: A method for preventing resynchronization in the ieee $802.11 \mathrm{p}$ standard. In 2015 IEEE Symposium on Computers and Communication (ISCC), pages 457-462. IEEE, 2015.

20. C. Anastasiades, J. Weber, and T. Braun. Dynamic unicast: Information-centric multi-hop routing for mobile ad-hoc networks. Computer Networks, 2016.

21. E. Baccelli, C. Mehlis, O. Hahm, T. Schmidt, and M. Wählisch. Information centric networking in the iot: Experiments with ndn in the wild. In 1st ACM Conference on Information-Centric Networking (ICN-2014). ACM, 2014.

22. C. Yi, A. Afanasyev, L. Wang, B. Zhang, and L. Zhang. Adaptive forwarding in named data networking. $A C M$ SIGCOMM computer communication review, 42(3):62-67, 2012.

23. A. Udugama, X. Zhang, K. Kuladinithi, and C. Goerg. An on-demand multi-path interest forwarding strategy for content retrievals in ccn. In 2014 IEEE Network Operations and Management Symposium (NOMS), pages 1-6. IEEE, 2014

24. J. Son, D. Kim, H. S. Kang, and C. S. Hong. Forwarding strategy on sdn-based content centric network for efficient content delivery. In 2016 International Conference on Information Networking (ICOIN), pages 220-225. IEEE, 2016.

25. S. Charpinel, C. A. S. Santos, A. B. Vieira, R. Villaca, and M. Martinello. Sdccn: A novel software defined content-centric networking approach. In 2016 IEEE 30th International Conference on Advanced Information Networking and Applications (AINA), pages 87-94. IEEE, 2016.

26. D. H. Lee, K. Thar, D. Kim, and C.S. Hong. Efficient parallel multi-path interest forwarding for mobile user in ccn. In 2016 International Conference on Information Networking (ICOIN), pages 390-394. IEEE, 2016.

27. A. Gomes and T. Braun. Load balancing in lte mobile networks with information-centric networking. In 2015 IEEE International Conference on Communication Workshop (ICCW), pages 1845-1851. IEEE, 2015.

28. S. A. Marandi, T. Braun, K. Salamatian, and N. Thomos. A push-based content advertisement approach for information-centric networks. 2016.

29. Y. T. Yu and M. Gerla. Information-centric vanets: A study of content routing design alternatives. In 2016 International Conference on Computing, Networking and Communications (ICNC), pages 1-5, Feb 2016.

30. C. Bernardini, T. Silverston, and O. Festor. Mpc: Popularity-based caching strategy for content centric networks. In 2013 IEEE International Conference on Communications (ICC), pages 3619-3623, June 2013.

31. S. B. Lee, S. H. Y. Wong, K. W. Lee, and S. Lu. Content management in a mobile ad hoc network: Beyond opportunistic strategy. In INFOCOM, 2011 Proceedings IEEE, pages 266-270, April 2011.

32. J. Iqbal and P. Giaccone. Interest-based cooperative caching in multi-hop wireless networks. In 2013 IEEE Globecom Workshops (GC Wkshps), pages 617-622, Dec 2013.

33. L. Gallos, S. Havlin, M. Kitsak, F. Liljeros, H. Makse, L. Muchnik, and H. Stanley. Identification of influential spreaders in complex networks. Nature Physics, 6(11):888-893, Aug 2010.

34. C. Bernardini, T. Silverston, and O. Festor. Sociallyaware caching strategy for content centric networking. In Networking Conference, 2014 IFIP, pages 1-9, June 2014.

35. G. Moualla, P. A. Frangoudis, Y. Hadjadj-Aoul, and S. Ait-Chellouche. A bloom-filter-based socially aware scheme for content replication in mobile ad hoc networks. In 2016 13th IEEE Annual Consumer Communications Networking Conference (CCNC), pages 359-365, Jan 2016.

36. D. Katsaros and P. Basaras. Detecting Influential Nodes in Complex Networks with Range Probabilistic Control Centrality, pages 265-272. Springer International Publishing, Cham, 2015.

37. J. Kangasharju, J. Ott, and O. Karkulahti. Floating content: Information availability in urban environments. In Pervasive Computing and Communications Workshops (PERCOM Workshops), 2010 8th IEEE International Conference on, pages 804-808, March 2010.

38. E. Hyyti, J. Virtamo, P. Lassila, J. Kangasharju, and J. Ott. When does content float? characterizing availability of anchored information in opportunistic content sharing. In INFOCOM, 2011 Proceedings IEEE, pages 31373145, April 2011 